\title{
Measurement of tendon excursion velocity with colour Doppler imaging: a preliminary study on flexor pollicis longus muscle
}

\author{
Bülent S. Clgalı ${ }^{\mathrm{a}, \mathrm{b}}$, H. Muzaffer Buyruk* ${ }^{* a}$, Christiaan J. Snijders ${ }^{\mathrm{c}}$, Johan S. Laméris ${ }^{\mathrm{d}}$, \\ Wim P.J. Holland ${ }^{\text {e, Recep Mesut }}{ }^{\mathrm{b}}$, Hendrik J. Stam ${ }^{\mathrm{a}}$ \\ 'Institute of Rehabilitation, University Hospital Rotterdam, Dr. Molewaterplein 40,3015 GD Rotterdam, The Netherlands \\ 'Department of Anatomy, Medical Faculty of Trakya University, Edirne, Turkey \\ 'Department of Biomedical Physics and Technology, Erasmus University Rotterdam, Dr. Molewaterplein 50, 3000 DR Rotterdam. \\ The Netherlands \\ 'Department of Radiology, University Hospital Rotterdam, Dr. Molewaterplein 40, 3015 GD Rotterdam, The Netherlands \\ 'Central Instrumentation Department, Erasmus University Rotterdam, Dr. Molewaterplein 50, 3000 DR Rotterdam, The Netherlands
}

Received 24 January 1996; revised 26 January 1996; accepted 16 February 1996

\begin{abstract}
Purpose: To study the use of colour Doppler imaging (CDI) for the measurement of maximum and mean tendon velocity. Recent studies showed that CDI, normally used for blood flow examinations, can be used for the imaging of tendons at the hand and wrist region. Although other modalities are available for imaging of the musculo-skeletal system, in vivo measurements of the velocity of tendon excursion are not possible. Methods: The flexor pollicis longus (FPL) tendon of 16 healthy volunteers was measured bilaterally at two levels (wrist and thenar). A splint from the fingers along the proximal lower arm was applied. The thumb was fixed to the splint from the first phalanx to allow flexion of the interphalangeal (IP) joint only. Pulsed CDI was used for the measurements. The maximum and mean velocities of the FPL tendon were measured at spectrum display mode during continuous voluntary contractions. At least 10 sequential Doppler peaks $(\mathrm{cm} / \mathrm{s})$ were recorded at every trial. The measurements were repeated three times. Paired $t$-test and correlation coefficients were calculated between levels on the same side and the opposite side. Results: No significant differences were found between two levels of the same hand and of the opposite hand. As expected, the data revealed variations in the inter-individual tendon velocities. Conclusions: The velocity of the excursion of the FPL tendon can be measured with CDI with good reproducibility. It is expected that velocity measurements can be used in the future for the assessment of other tendons affected by various disorders.
\end{abstract}

Keywords: Hand, tendon speed measurements; Ultrasound (US), Doppler studies

\section{Introduction}

Maximum tendon excursion velocity depends on the condition of the tendon, the tendon sheath and the muscle belly and its innervation. Intrinsic and extrinsic muscle diseases and aging [1] cause a significant decrease in the maximal velocity of isotonic muscle contraction. Dynamometers can determine this phenomenon indirectly by measuring joint rotation velocity and exerted moments of force. This gives optimal results for single joint motions driven by one muscle, as in knee extension. In case of multiple joints and/or muscles it is more

\footnotetext{
* Corresponding author.
}

complex to measure the displacement velocity of a specific tendon. Specific maximum velocity measurements for tendons such as hand flexors, may be valuable for diagnosis, treatment and follow-up.

The power $(P)$ output of a muscle is the product of muscle (tendon) force $(F)$ and tendon velocity $(v)$ :

$P=F \cdot v\left(N m . s^{-1}\right)$

The acceleration $(a)$ of a tendon excursion depends on the mass $(m)$ that must be propulsed by the given muscle force $(F)$ :

$$
a=F / m\left(m . s^{-2}\right)
$$


The non-invasive accurate measurement of in vivo muscle and tendon velocity or the amount of excursion is not possible with the available techniques. The musculo-skeletal system can be imaged by using different techniques $[2,3]$, including radiography, ultrasound [4,5], CT scan [6,7], and MRI. However, these techniques have the disadvantage that they fail to give quantitative information about the movements of muscles and tendons. Although the non-invasive examinations with superficial EMG [8] and muscle dynamometry $[9,10]$ are closely related to the function, it is hard to isolate individual muscle function. With grey scale ultrasound such functions can be visualised, but not quantified.

For the diagnosis of cardiac and vascular disorders sonography and the colour display of Doppler, shifts from moving reflectors are often used [11]. Similar to such blood flow examinations we demonstrated that colour Doppler imaging (CDI) can visualise muscle and tendon movements [12] and differences due to pathology [13]. The purpose of the present study is to show whether the non-invasive quantification of tendon velocity is possible with CDI and to test the reproducibility of tendon velocity measurements which will be executed on healthy volunteers.

\section{Materials and methods}

Maximum and mean velocity was measured with a CDI on the flexor pollicis longus (FPL) tendon. Sixteen healthy right-handed volunteers (aged 24-44, mean 33.3 years) participated in the study. A dorsal lower arm splint, made of Aquaplast, from fingertips to elbow was applied in order to immobilise the lower arm. The thumb was fixed, by a Velcro hook and loop attachment band, to the splint from the first phalanx to only allow flexion of the interphalangeal (IP) joint. A Philips AD1 (Angio Dynagraph 1), with software version 2.5, pulsed multi-channel CDI scanner was used. A procedure similar to blood flow examinations was followed. A 7.5MHz linear transducer was used. An adjustment button (threshold) allowed to determine the best sensitivity for the apparatus to display slow movements. The location of the FPL muscle was determined with the help of the threshold button. An $18^{\circ}$ wedge between the probe and the skin was used.

The measurement procedure was standardized as follows. The excursion of the FPL tendon was measured at two levels; the thenar and the wrist (distal radius). The exact measurement points were marked on the skin. Volunteers were asked to flex and extend their thumbs continuously with maximal speed. After a clear Doppler image of the excursion had been obtained in the threshold mode, the apparatus was switched to the spectrum mode (Fig. 1). The angle between the ultrasound and the tendon was corrected with the angle correction

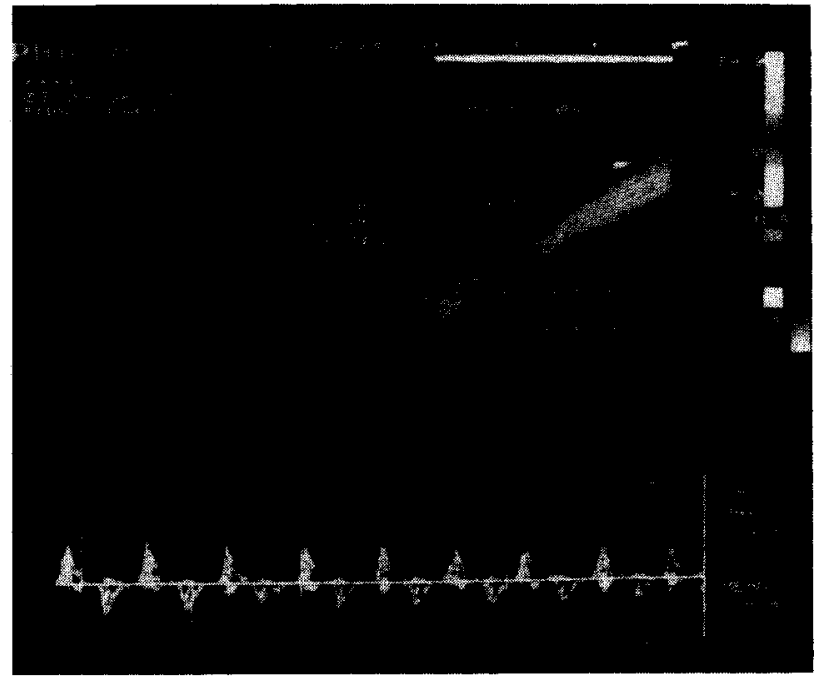

Fig. 1. A typical CDI image of FPL tendon right hand thenar level.

option placed on the apparatus. The maximum and mean of the point velocity options were chosen from the spectral menu on the screen. With the maximum velocity selected, the system overlays the maximum Doppler shifted frequency on the spectral waveform. The maximum velocity is coloured green so as to be differentiated from the spectral waveform and is a scrolling waveform along with the spectra. The maximum Doppler shift is defined as the frequency below which $95 \%$ of the frequencies fall. The apparatus then calculates the velocity values from the equation of Doppler shift and displays it on the screen. It can also calculate the mean velocity at the same time. The mean Doppler shift is defined as the centroid frequency. The maximum and mean velocities of the FPL tendon were measured in the spectrum display mode during continuous active movement. At least 10 sequential Doppler peaks $(\mathrm{cm} / \mathrm{s})$ were recorded at every measurement. The measurements were repeated three times bilaterally.

The measurement results were processed with a spreadsheet and a statistics program. The coefficient of variance (C.V.) of the repeated measurements for each level was calculated separately for each subject. The C.V. of the measurements from all of the subjects were calculated from the average of all the measurements for each level. Paired $t$-test and correlation were used to demonstrate if there were any significant differences between the measurements of the levels and the sides. All statistical functions were applied to both measurement results of maximum and mean Doppler velocities. For statistical significance a level of $P<0.05$ was chosen.

\section{Results}

It was possible to measure the FPL maximum and mean displacement velocity in all subjects at all mea- 


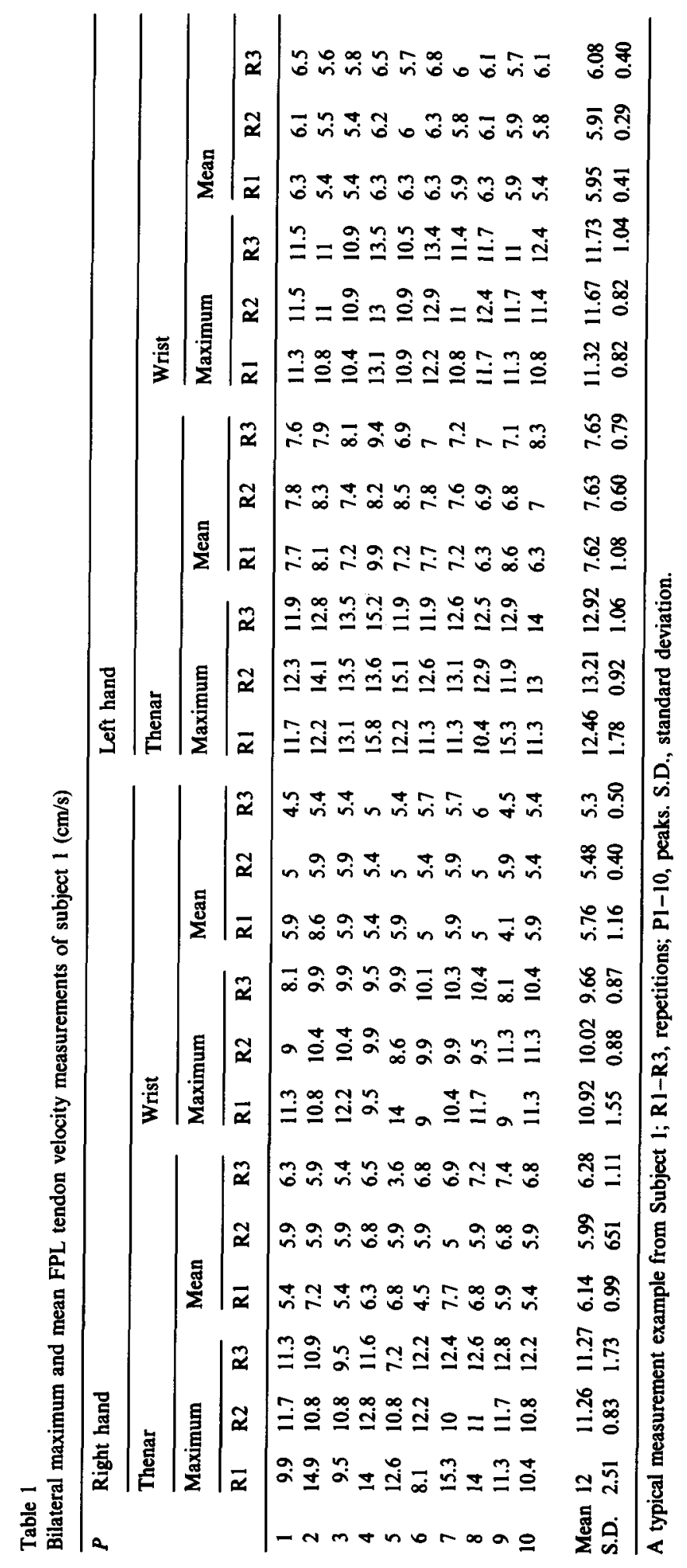


Table 2

Mean of coefficient of variation (\%)

\begin{tabular}{llllll}
\hline \multirow{2}{*}{ Site } & \multicolumn{2}{l}{ Maximum velocity } & & \multicolumn{2}{l}{ Mean velocity } \\
\cline { 2 - 3 } \cline { 5 - 6 } & Peaks & Repetitions & & Peaks & Repetitions \\
\hline LHTL & 12 & 5 & 12 & 7 \\
LHWL & 12 & 6 & 11 & 6 \\
RHTL & 10 & 6 & 10 & 6 \\
RHWL & 11 & 7 & & 11 & 7 \\
\hline
\end{tabular}

Mean coefficient of variation of peaks and repetitions of maximum and mean velocity. LHTL, left hand thenar level; LHWL, left hand wrist level; RHTL, right hand thenar level; RHWL, right hand wrist level.

surement sites with CDI. Doppler waves appeared as shown in Fig. 1. Table 1 presents the typical measurement results of one subject. Subject 2 displayed the highest averages of maximum velocities $17.77 \pm 1.36$ $\mathrm{cm} / \mathrm{s}, 17.37 \pm 2.20 \mathrm{~cm} / \mathrm{s}, 15.43 \pm 0.95 \mathrm{~cm} / \mathrm{s}$ from the left hand thenar level (LHTL), left hand wrist level (LHWL), and right hand thenar level (RHTL), respectively. Subject 16 displayed the highest velocity from the right hand wrist level (RHWL) $14.80 \pm 1.22 \mathrm{~cm} / \mathrm{s}$. The lowest velocities were recorded from LHTL $9.22 \pm 0.64$ $\mathrm{cm} / \mathrm{s}$ of subject 13 , from LHWL and RHTL $10.6 \pm 0.23$ $\mathrm{cm} / \mathrm{s}$ and $8.41 \pm 0.72 \mathrm{~cm} / \mathrm{s}$, respectively, from subject 4 , from RHWL $9.76 \pm 0.35 \mathrm{~cm} / \mathrm{s}$ of subject 5 . C.V. of maximum and mean velocity measurements appeared to be high (low accuracy) for 10 sequential wave peaks, but was low (high accuracy) for measurement repetitions (Table 2). The intra-individual differences between thenar and wrist and between right and left were statistically significant. The correlation coefficients were high and ranged between 0.60 and 0.82 (Table 3).

\section{Discussion}

The FPL was selected because the tendon is superficial and therefore easy to image with CDI. Furthermore, it is the only flexor of the distal phalanx of the thumb and there are in general no inter-tendinous connections [14]. Some exceptions have been reported, such as anomalous tendon slips from the FPL to the deep flexor finger muscle which restricts FPL excursion [15].
FPL tendon maximum and mean velocities could be measured in all subjects. During fast thumb flexion/extension the maximum velocity was different for each repetition. We assume that these differences were caused by disoriented movements of the distal phalanx of the thumb. The frequency and the velocity of this movement do not only depend on muscular functions but also on neurological control during continuous voluntary movements. The assessment of the minimum number of repetitions necessary for the determination of a representative average is important for the design of future experiments. It is concluded that 10 repetitions are sufficient because $\mathrm{CV}$ is low, indicating good reproducibility. The correlation between the velocity measurements at thenar and wrist was high which indicates that different measurement sites with different tendon section, tissue thickness, and density do not affect the results. Measurements of the left and the right FPL were correlated less, which can be attributed to factors as central or peripheral neural control. From the results it can be concluded that if a series of 10 waves is recorded one time at one site, the maximal tendon excursion velocity is measured with high accuracy.

It is more difficult to obtain clear wave forms from the wrist area since the FPL muscle belly begins at the proximal carpal level and in the image it is displayed as a larger stripe in the CDI image. However, we observed no differences between the measurements of the maximum velocity at different sites of the same hand.

Although the maximum FPL velocity was slightly higher on the dominant side, the difference was not significant. A higher FPL maximum velocity measurement may be expected at the dominant side because of a higher skill than that at the non-dominant side. In addition to thumb flexion/extension, the subjects were asked to reach the full range of motion without using the accompanying muscles. In an earlier unpublished study we observed that a higher maximum velocity of FPL was easier obtained when the thumb was flexed and extended with intermediate pauses rather than with continuous flexion/extension.

The peak force and power output (and thus, indirectly, tendon velocity) of a muscle depend on numerous factors [16] such as: (a) muscle and fibre size and length; (b) architecture, such as the angle and physical proper-

Table 3

Correlation and paired $t$-test

\begin{tabular}{|c|c|c|c|c|c|c|c|c|}
\hline \multirow[t]{2}{*}{ Site } & \multicolumn{4}{|c|}{ Maximum velocity } & \multicolumn{4}{|c|}{ Mean velocity } \\
\hline & Mean & S.E. & $P$-value & $r$ & Mean & S.E. & $P$-value & $r$ \\
\hline LHTL & 0.2 & 0.33 & 0.54 & 0.81 & 0.41 & 0.16 & 0.02 & 0.82 \\
\hline LHWL & -0.76 & 0.89 & 0.4 & 0.74 & 0.38 & 0.2 & 0.07 & 0.71 \\
\hline RHTL & 1.31 & 1.14 & 0.26 & 0.67 & 0.18 & 0.25 & 0.49 & 0.60 \\
\hline RHWL & 0.34 & 0.35 & 0.35 & 0.71 & 0.15 & 0.18 & 0.42 & 0.71 \\
\hline
\end{tabular}

Paired $t$-test of mean velocity differences. 
ties of the fibre-tendon attachment and the fibre to muscle length ratio; (c) fibre type; (d) number of cross-bridges in parallel; (e) force per cross bridge; (f) peak $\mathrm{d} F / \mathrm{d} t$ (peak level of force development) N/s; (g) force velocity relationship; (h) fibre maximum velocity; (i) force-p and calcium relationship; and (j) the forcefrequency relationship. It is clear that if some of these components change due to any reason, such as a trauma which affects the first and second factor, the power output and the shortening velocity will also be affected.

The fibre maximum velocity can be measured by a slack test in vitro, but the single muscle shortening velocity or single tendon excursion velocity has not been previously measured directly from the tendons in vivo. In pathological situations in which the factors mentioned above are changed, tendon velocity measurements may be valuable to evaluate the impaired muscle condition. During our experiments, the average FPL maximal displacement velocity was measured. These measurements are valuable for relative changes, such as the difference between healthy and pathologic extremity. The values obtained from the apparatus are not calibrated to real displacement velocity of FPL tendon. Since the exact software of the apparatus was not available, filters and post-measurement calculations are unknown. It will be useful to compare in vivo measurements of maximum velocity the apparatus with phantom models in which tendon displacement velocity is precisely known and can be manipulated.

\section{Conclusion}

This study is the first to demonstrate that the velocity of single muscle contraction can be measured noninvasively with CDI; the difference in FPL tendon velocity between the wrist and thenar levels was nonsignificant; 10 repetitions of thumb flexion/extension deliver an accurate measurement of maximal FPL tendon velocity; FPL tendon velocity varies considerably between healthy subjects.

\section{References}

[1] Aoyagi Y, Shephard RJ. Aging and muscle function. Sports Med 1992; 14: 376-396.

[2] Mann FA, Wilson AJ, Gilula LA. Radiographic evaluation of the wrist: what does the hand surgeon want to know? Radiology 1992; 184: 15-24.

[3] Wilson AJ, Mann FA, Gilula LA. Imaging the hand and wrist. J Hand Surg 1990; 15B: 153-167.

[4] McGeorge DD, McGeorge S. Diagnostic medical ultrasound in the management of hand injuries. J Hand Surg 1990; 15B: 256-261.

[5] Dias JJ, Hui ACW, Lamont AC. Real time ultrasonography in the assessment of movement at the site of a scaphoid fracture non-union. J Hand Surg 1994; 19B; 498-504.

[6] Hoglund $M$, Tordai $P$, Engvist $O$. Ultrasonography for the diagnosis of soft tissue conditions in the hand. Scand $\mathrm{J}$ Reconstr Hand Surg 1991; 25: 225-231.

[7] James SE, Richards R, Mc Grouther DA. Three-dimensional CT imaging of the wrist. J Hand Surg 1992; 17B: 504-506.

[8] Buchanan TS, Moniz MJ, Dewald PA, Rymer WZ. Estimation of muscle forces about the wrist joint during isometric tasks using an EMG coefficient method. J Biomech 1993; 26: $547-560$.

[9] Moritani T. Neuromuscular adaptations during the acquisition of muscle strength, power and motor tasks. J Biomech 1993; 26: 95-107.

[10] Fitts RH, McDonald KS, Schulter JM. The determinants of skeletal muscle force and power: their adaptability with changes in activity pattern. J Biomech 1991; 24: 111-122.

[11] Kisslo J, Adams DB, Belkin RN. Doppler colour flow imaging. New York: Churchill Livingstone, 1988.

[12] Stam HJ, Buyruk HM, Laméris JS, Snijders CJ. Colour Doppler ultrasound in dynamic imaging of the musculo-skeletal system. J Rehabil Sci 1994; 7: 49-52.

[13] Buyruk HM, Stam HJ, Laméris JS, Schut HA, Snijders CJ. Colour Doppler ultrasound examination of hand tendon pathologies: a preliminary report. J Hand Surg (in print).

[14] Williams PL, Warwick R, Dyson M, Bannister LH. Gray's Anatomy. 37th ed. Edingburgh: Churchill Livingstone, 1992.

[15] Leijnse JNAL, Snijders CJ, Bonte JE, Landsmeer JMF, Kalker JJ, van Der Meulen JC, Sonneveld GJ, Hovius SER. The hand of musician: the kinematics of the bidigital finger system with anatomical restrictions. J Biomech 1993; 26: 1169-1179.

[16] Fitts RH, McDonald KS, Schulter JM. The determinants of skeletal muscle force and power: their adaptability with changes in activity pattern. J Biomech 1991; 24: 111-122. 